\title{
Operations Research, Mathematics, Computer Science and Statistics: The Relationships
}

\author{
Adewoye S. Olabode
}

Department of Mathematics, Yaba College of Technology, Yaba, Lagos, Nigeria

\begin{abstract}
Many people find it hard seeing any difference between Mathematics, Operations Research, Statistics, Computer Science and other disciplines while others are just plain confused. In this work, OR and its applications are being exposed and then compared in order to look into the relationships between OR, Mathematics, Computer Science, Statistics and other fields. It has been realized that all these areas of knowledge are also interrelated with other areas such as Engineering, Physics, Microbiology, Economics etc.
\end{abstract}

Keywords: Mathematics, Operations Research (OR), Statistics, Computer Science, Mathematical Models.

Received 10 July 2019 | Revised 28 November 2019 | Accepted 29 January 2020

\section{Introduction}

Many people find it hard seeing any difference between Operations Research, Mathematics, Computer Science, Statistics, and other disciplines, while others are just plainly confused. This paper will in some way or another show the relationships between Operations Research, Mathematics, Computer Science and Statistics to other subjects and hence eliminating confusions. An interesting area of Applicable Mathematics known as "Operational Research". This wonderful field of specialization combines Mathematics, Statistics, Computer Science, Physics, Engineering, Economics, and Social Sciences. It plays an important role in solving real-world business problems. Numerous companies, industries and government agency requires Operations Research professionals to apply mathematical models to a wide range of many challenging problems [3].

The term 'Operational Research' is commonly used in United Kingdom and European countries whereas, in the United State of America (USA), it is known as "Operations Research". Both terms means the same thing. It is often shortened as "OR". Other terms used for OR are: "Management

\footnotetext{
*Corresponding author at: Department of Mathematics, Yaba College of Technology, Yaba, Lagos, Nigeria

E-mail address: adewoye2012@gmail.com
} 
Science", "Industrial Engineering" and "Decision Sciences" to mention just few. The manifoldness of names comes primarily from the different academic departments that have hosted courses in this field. In many universities, there has always been conflict of which department should house Operations Research. The competing departments are mathematics department, statistics department, computer department, business administration department and system engineering department.[3],[12],[14]

"Operations research" and "management science" are terms that are used interchangeably to describe the discipline of using advanced analytical techniques to make better decisions and to solve problems. The procedures of operations research were first formalized by the military. They have been used in wartime to effectively deploy radar, search for enemy submarines, and get supplies to where they are most needed. In peacetime and in private enterprises, operations research is used in planning business ventures and analyzing options by using statistical analysis, computer modeling, linear programming, and other mathematical techniques.

Large organizations are very complex. They must effectively manage money, materials, equipment, and people. Operations research analysts find better ways to coordinate these elements by applying analytical methods from Mathematics, Science, and Engineering. Analysts often find many possible solutions for meeting the goals of a project. These potential solutions are presented to managers, who choose the best course of action in attacking the problem.[3],[5],[6].

Operations research analysts are often involved in top-level strategizing, planning, and forecasting. They help to allocate resources, measure performance, schedule, design production facilities and systems, manage the supply chain, set prices, coordinate transportation and distribution, or analyze large databases.

\section{Methodology of Operations Research}

a. Problem Definition and Objectives (motivation, objectives, decision variables, control parameters, constraints);

b. Construction of a Mathematical Model (representation of complex systems by analytical or numerical models, relationships between variables, performance metrics);

c. Data Collection (model inputs, system observations, validation, tracking of performance);

d. Derivation of a Solution from the Model (simulation, stochastic processes, optimization, heuristics, and other mathematical methods);

e. Testing the Models and its Solution (model testing, sensitivity analysis, model robustness);

f. Establishing control (sensitivity analysis which serves as a guide to the variable when altered and make a significant change to the solution);

g. Interpretation and Implementation (solution ranges, trade-offs, visual or graphical representation of results, decision support systems). 
The implementation stage is obviously very important stage in any OR project. In majority of cases the implementation is not carried out by the OR team but by personnel already working in the area. These personnel should have been involved with the project throughout and should be sufficiently acquainted with what was going on to be able to identify with the project. The solution to the problem has to be translated into set of operating instructions which can be handled by the personnel involved. To carry out the above steps, a good foundation in Mathematics and familiarity with other disciplines (such as Statistics, Computer, Physics, Economics, Business Administration, Microbiology, Engineering, etc.), as well as clear thinking and intuition is essential. The mathematical sciences foundation prepare "who be analyst" to apply tools and techniques of OR and use a logical process to analyze and solve problems.

\section{OR and its Applications}

OR became a confirmed field during World War II. The British were the first to organize formal operations research groups. In 1939, the G.A. Roberts and Dr. E.C. Williams independently developed early warning communication system studies for the Royal Air Force. Mathematical models were developed to determine the most effective use of radar and other new defense technologies at the time. The group was later given the name Anti- Aircraft Command Research Group. OR groups were thereafter formed in the U.S. to meet needs of wartime operations, such as the optimal movement of troops, supplies, and equipment.[3],[7],[10],[12]

After the Second World War, applications of OR turned to peaceful means. OR departments now exist in industry, military, government, and academia all over the world. Examples of areas of successful applications of OR in recent years include the following:

a. Airline Industry (routing and flight plans, crew scheduling, revenue management);

b. Telecommunications (network routing, queue control);

c. Manufacturing Industry (system throughput and bottleneck analysis, inventory control, production sequencing and scheduling, capacity planning);

d. Healthcare management systems (hospital management, facility design);

e. Transportation systems (traffic control systems, logistics, network flow, airport terminal layout, air ticketing and reservation location planning, integrated transportation models,);

f. a complex project which affect the overall duration of the project;

g. Designing the layout of a factory for efficient flow of materials;

h. Constructing a telecommunications network at low cost while still guaranteeing QoS (quality of service) or QoE (Quality of Experience) if particular connections become very busy or get damaged;

i. Road traffic management and 'one way' street allocations i.e. allocation problems;

j. Determining the routes of school buses (or city buses) so that as few buses are needed as possible; 
k. Designing the layout of a computer chip to reduce manufacturing time (therefore reducing cost);

1. Managing the flow of raw materials and products in a supply chain based on uncertain demand for the finished products;

m. Efficient messaging and customer response tactics;

n. Robotizing or automating human-driven operations processes and artificial intelligence;

o. Globalizing operations processes in order to take advantage of cheaper materials, labor, land or other productivity inputs;

p. Managing freight transportation and delivery systems;

q. Scheduling namely: personnel staffing, manufacturing steps, project tasks, sports events and their television coverage.

\section{Mathematics and Operations Research}

Many mathematical techniques and solutions were developed specifically for OR applications. These techniques and solutions arose from basic mathematical ideas and became major areas of expertise for solving operational problems.

Optimization. Many problems in our rapidly changing world require finding the maximum or minimum of an objective function of a set of decision variables, subject to a set of constraints on those variables. We either maximum profit, minimum cost, or minimum delay and other measures of effectiveness. Most time, there are several decision variables and the solution is not obvious. Use of mathematical programming for optimization will be of great assistance in such situations. Some of these techniques include: Linear programming model, non-linear programming, integer programming, stochastic programming and dynamic programming.

Analysis of stochastic processes. Processes that are random are known as stochastic processes. They relied on results from applied probability and statistical modeling. Most of the real-world problems involve uncertainty, and mathematics has been playing important role in identifying ways to manage such problems. Modeling uncertainty is extremely important in risk analysis involving complex systems, such as nuclear power generation, space shuttle flights, large dam operations, space shuttle flights and emergency rescue operations of victims of natural disaster.

Some of the topic of stochastic processes is queuing theory, Markov process. A queuing process consists customers arriving at a service facility, then waiting in line (queue if all servers are busy, eventually receiving service and finally departing from the facility). A common example is the single-server queue in which customer arrivals and service times are random. Figure 1 illustrates the queue. Mathematical analysis has been essential in understanding queue behavior and quantifying impacts of decisions. Equations have been derived for the queue length, waiting times, and probability of no delay, and other measures. For more on how such equations are developed see [3],[5],[6]. Some areas where applications of queuing models have been 
successfully applied includes:, customers waiting to be served in a bank or supermarket checkout, cargo loading/ unloading in the port, emergency medical aid for people needing ambulances, students at registration center, orders waiting for production, ships docking at a harbor, users of the internet, and customers served at a restaurant.

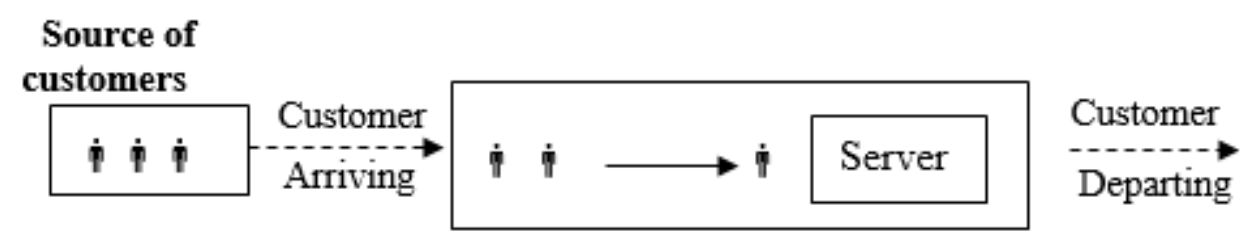

Figure 1. Single queue

Performance analysis of a production line in a manufacturing concern. A production line consists of some workstations that perform various tasks, called operations. Jobs flow through the production line to be processed at each station. Buffers between stations hold the output of one station and allow it to wait as input to the next. A finite buffer can fill and block output from an upstream station or can empty and starve a downstream station for input. Blocking and starving are key mechanisms of the complex interactions between queues that form in the line. Mathematical modeling is used to capture the impact on throughput of station reliabilities, as well as processing rates and buffer sizes. A model can support operating decisions, such as how to improve a line to meet a throughput target, how to identify bottlenecks, and how much buffer space to allocate in line design.

Transportation, assignment and network model. Transportation problems are concerned with the distribution of a certain product from several sources to numerous localities at minimum cost. To achieve this the transportation algorithm (TA) must be followed, that is: Find the initial solution; test the solution for optimality; improve the solution if not optimal; repeat step two and step three until optimality is attained. The methods of finding initial solution (North west corner rule, least cost method, the Vogel's approximation and Russell's method) represents the first step in solving transportation problems. Figure 2 below shows the TA. Assignment model is essentially, how to assign $\mathrm{n}$ items to $\mathrm{n}$ other items on a one- to- one basis in such a way that some measures of effectiveness is optimized. Application area of assignment can be seen in: assigning sales territories, crime prevention, military combat effectiveness, and personnel job assignments. Network analysis is the application of systematic reasoning for planning, scheduling and controlling practical solutions where many separate task are involved.[3] For a network of origins and destinations, there are many shipping alternatives, including choices of transportation mode (e.g., road, rail, air) and geographical routes. Some key decisions are routing options over the network, and shipping frequencies on network links. As shown in Figure 3, routing options involve shipping direct, via a terminal or distribution center, and by a combination of routes. Trade-offs between these costs are complex for large networks, and finding the optimal solution 
is a challenging mathematical problem. In addition to decisions for operations of a given network, there are major strategic decisions, such as the selection and location of distribution centers.

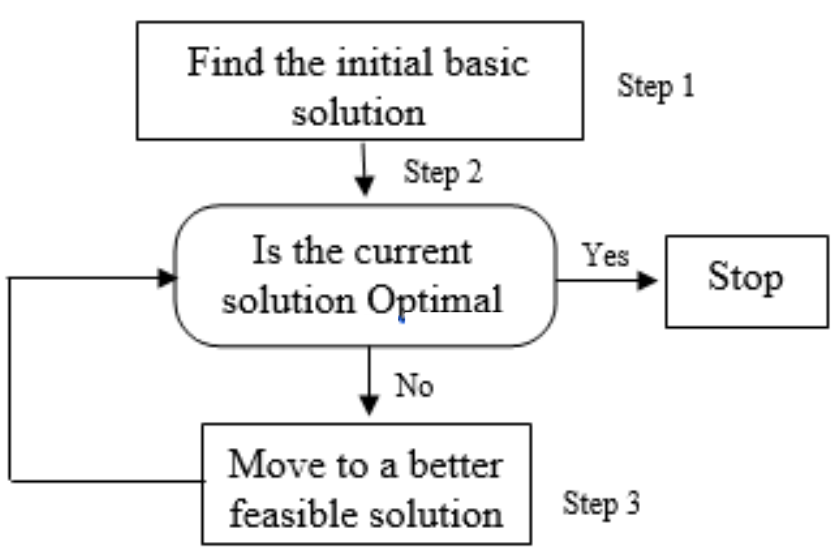

Figure 2. The transportation algorithm (TA)

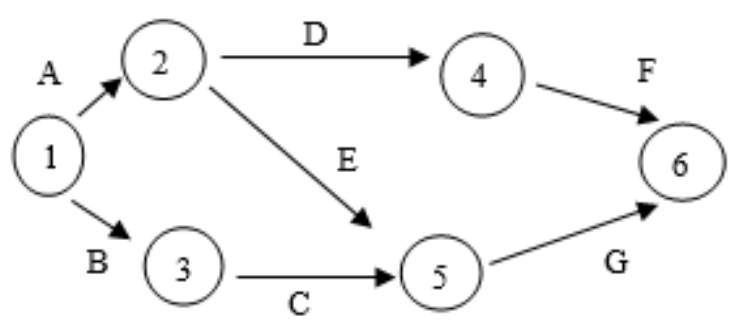

Figure3. Network Routing Options

Inventory control. An inventory is the stock of any item kept to contribute to an organization's output. Inventory may be either a physical product or a service. The primary objective of inventory is to meet demand at minimum cost. This involves the assignment of appropriate values to the decision variables of when to procure, how much to procure, and from what source to procure by constructing and optimizing a mathematical model. At optimum level there are four critical control levels being used to maintain stock (minimum stock level, maximum stock level, re- order level and average stock). All these control level involves mathematical computations. There several other OR techniques requiring mathematical techniques. We have Location planning (where best to locate the hub to serve markets with minimal travel distances), and facility layout (how best to design airport terminals, bus terminals, etc., to minimize walking distances, maximize number of gates, allow for future growth).

In general, Operations Research requires use of mathematics to model complex systems, analyze system, identify robust approach to solve the problem if available, and develop decision support tools. Mathematical models are mathematical statements of the facts of a situation. In a rapidly changing world, a lot complexity come with it and the need to dependent more on new technology, mathematics, computer and statistics to solve the problems. 


\section{Relationship Between Mathematics and Statistics}

By examining the methodologies of these two related subjects. Basically, the mathematical method consists of the following two stages.

\section{Stage 1}

Proposes hypothesis i.e. provisional explanation of observed facts

\section{Stage 2}

Attempt to prove or disprove the proposed hypothesis using theorems and corollaries that have already been satisfactorily tested.

The statistical method has three main stages namely:

\section{Stage 1}

The collection of data: facts and figures

\section{Stage 2}

The numerical statistic stage, which adopts the following approach in order to process the facts and figures so that deductions and inferences can be made from them:

a. Propose a hypothesis about a problem situation, such a hypothesis is usually directed towards a specific set of data (i.e. population)

b. Draw a random sample from the population

c. Accept or reject the hypothesis according to the information obtained from (a)

\section{Stage 3}

Use mathematical statistics to give theoretical support and justification to the techniques utilized in stage 2 .

\section{The Relationship}

Mathematics and Statistics are used widely in many diverse disciplines. In operation research, for example, they provide a vehicle for objectivity, i.e., for expressing, analyzing and calculating results. The use of probability theory (which is a subset of statistics) plays a fundamental role in decision making under uncertainty. In such circumstances there is a need to take into consideration the intuitive, judgmental aspects which are related to the problem, as well as the scientific objectivity of Mathematics and Statistics.

\section{Computer Science and Operations Research}

OR and Computer Science have long been related. The principal motivation for the development of digital computers arose from their potential ability to some major real life problems. Some of the most computationally demanding problems are those to which OR techniques are applied. Without the digital computer, many of those techniques would be of little practical importance. Since 1952 when the first scale OR application was successfully accompanied on a digital computer, the use of computerized OR techniques and international environment, productivity is the key, and OR is an essential productivity tool. The relative inaccessibility difficulty of use, and 
expensive large computers limited the applications of Operations Research in the past. With the advent of personal computer, such problem has been overcome. The personal computer provides accessibility and it is easy to use. Thousands of organizations, both large and small are engaged in the development of computer software to assist in assigning, budgeting, distributing, financial planning, forecasting, inventory controlling, scheduling and many other activities. At the center of these are OR techniques.

a. OR needs vast amount of data to be summarized and analyzed. Computer does all these very fast and accurately.

b. OR does a great deal of trial and error in developing, modeling and each time a great deal of computation is required. Doing this manually is entirely slow. What would take many months or years will take computer a few minutes.

c. OR stores a lot of information on its developments. The computer does this accurately.[3],[13].

\section{Statistics and Operational Research}

Statistics is useful in OR for many reasons, some of these are;

a. OR needs quantitative information about the behavior, attributes and features of the systems under study and the entities involved in them.

b. Statistical analysis of data may suggest a different problem that requires to be tackled rather than what was earlier thought to be the one to be tackled. For example data analysis may suggest that bad debts pattern is the major problem of a bank rather than investment policy, or expenditure on infrastructure.

\section{Nature of Statistics in Operational Research}

This can be viewed in three angles:

a. Method of collection, organization and summarizing, presentation and analysis of data.

b. The actual data and population attributes derived from. For instances, individual employment data and trends obtained from them e.g. categories that are most prone to absenteeism, poor quality of work, etc.

c. Hypothesis testing in establishing relationships to be included in a Model.

\section{Operations Research Analyst}

OR analysts can model difficult practical problems and offer valuable solutions and policy guidance for decision-makers. Constraints involving budgets, capital investments, and 
organizational considerations can make the successful implementation of results as challenging as the development of mathematical models and solution methods.

Operations research analysts start a project by listening to managers describe a problem. Then, analysts ask questions and formally define the problem. For example, an operations research analyst for an auto manufacturer may be asked to determine the best inventory level for each of the parts needed on a production line and to ascertain the optimal number of windshields to be kept in stock. Too many windshields would be wasteful and expensive, whereas too few could halt production.

Analysts would study the problem, breaking it into its components. Then they would gather information from a variety of sources. To determine the optimal inventory, operations research analysts might talk with engineers about production levels, discuss purchasing arrangements with buyers, and examine storage-cost data provided by the accounting department.

Relevant information in hand, the analysts determine the most appropriate analytical technique. Techniques used may include a Monte Carlo simulation, linear and nonlinear programming, dynamic programming, queuing and other stochastic-process models, Markov decision processes, econometric methods, data envelopment analysis, neural networks, expert systems, decision analysis, and the analytic hierarchy process. Nearly all of these techniques involve the construction of a mathematical model that attempts to describe the system being studied. So, the problem of the windshields, for example, would be described as a set of equations that try to model real-world conditions.

The use of models enables the analyst to explicitly describe the different components and clarify the relationships among them. The descriptions can be altered to examine what may happen to the system under different circumstances. In most cases, a computer program is developed to numerically evaluate the model.

Usually the model chosen is modified and run repeatedly to obtain different solutions. A model for airline flight scheduling, for example, might stipulate such things as connecting cities, the amount of fuel required to fly the routes, projected levels of passenger demand, varying ticket and fuel prices, pilot scheduling, and maintenance costs. By assessing different possible schedules, the analyst is able to determine the best flight schedule consistent with particular assumptions.

Based on the results of the analysis, the operations research analyst presents recommendations to managers. The analyst may need to modify and rerun the computer program to consider different assumptions before presenting the final recommendation. Once managers reach a decision, the analyst usually works with others in the organization to ensure the plan's successful implementation. 
Work environment. Operations research analysts generally work regular hours in an office environment. However, because they work on projects that are of immediate interest to top managers, operations research analysts often are under pressure to meet deadlines and may work more than 40 hours a week.

College degree in operations research generally is required. Computer programming skills are essential.

Education and training. Employers generally prefer applicants with at least a master's degree in operations research or a closely related field — such as Computer Science, Engineering, Business Mathematics, Information Systems, or Management Science — coupled with a bachelor's degree in Computer Science or a Quantitative discipline such as Economics, Mathematics, or Statistics. Dual graduate degrees in Operations Research and Computer Science are especially attractive to employers. There are more than 130 programs in operations research and related studies in colleges and universities across the United States.

Continuing education is important for operations research analysts. Keeping up to date with technological advances and improvements in analytical methods is vital for maintaining their problem-solving skills.

Other qualifications. Computers are the most important tools used by operations research analysts, so analysts must have training and experience in programming. Analysts typically also need to be proficient in database collection and management, and the development and use of sophisticated software packages.

Operations research analysts must be able to think logically, work well with people, and write and speak well.

Advancement. Beginning analysts usually perform routine work under the supervision of more experienced analysts. As novices gain knowledge and experience, they are assigned more complex tasks and are given greater autonomy to design models and solve problems.

Operations research analysts can advance by becoming technical specialists or supervisors on more complicated projects. Analysts also gain valuable insights into the industry where they work and may assume higher level managerial or administrative positions. Operations research analysts with significant experience or expertise may become consultants, and some open their own consulting practice.

\section{Operations Research Societies}

The International Federation of Operational Research Societies (IFORS) is the coordinating organization of all operations research societies.[14]. Significant among these societies are: 
a. Institute for Operations Research and Management Sciences (INFORMS)

b. The Operational Research Society.

c. EURO is the association of European Operational Research Societies.

d. CORS is the Canadian Operational Research Society.

e. ASOR is the Australian Society for Operations Research. MORS is the Military Operations Research Society: based in the United States since 1966 with the objective of enhancing the quality and usefulness of military operations research analysis in support of defense decisions. (MORS)

f. ORSNZ is the Operations Research Society of New Zealand.

g. ORSP is the Operations Research Society of the Philippines.

h. ORSI the Operational Research Society of India.

i. ORSSA the Operations Research Society of South Africa.

j. IORMS is the Institute of Operations Research and Management Science of Nigeria.

\section{Conclusion}

This paper has been able to clarify some of the misconceptions people have towards the relationships between Operational Research, Mathematics, Statistics, Computer Science and other relevant fields. We have been able to show the interrelationships between Operational Research, Mathematics, Statistics And Computer Science as well as different mathematical models and their applications.

\section{REFERENCES}

[1] Adewoye, S.O.(1994): Policy iteration methods for Markov and semi-Markov decision Processes University of Ibadan, Ibadan.

[2] Adewoye, S.O. (2004): Basic Statistics for Engineering, Economics and Management, Ojo Press, Lagos.

[3] Adewoye, S.O. (2004): Introduction to Operational Research, Deto Press, Lagos.

[4] Allen, A. O. (1990). Probability, Statistics, and Queuing Theory - With Computer Science Applications (Second Edition). Academic Press, Orlando, Florida.

[5] Anderson M.Q. and Lievano R.J.(1986): Quantitative Management An Introduction, Kent Publishing, $2^{\text {nd }}$ ed , Boston

[6] Anderson, D.R; Sweeney, D.J.; and Williams, T.A (1984): An introduction to management Science: West Publishing.

[7] Charles R.S. (2006): History of Operations Research in the United States Army vol1. 1942 -1962. History.army.mil/ html/books/his-op-research.

[8] Frederick S. Hillier \& Gerald J. Lieberman (2005) Introduction to Operations Research, McGraw-Hill: Boston MA; 8th. (International) Edition

[9] Hamdy A. Taha (2006): Operations Research: An Introduction, Prentice Hall; 8th. Edition

[10] Hillier, F. S. and Lieberman, G. J. (2001): Introduction to Operations Research (Seventh Edition). McGraw-Hill, New York. 
[11] Larson, R. C. and Odoni, A. R. (1981): Urban Operations Research. Prentice-Hall, Englewood Cliffs, New Jersey.

[12] Thomas William (2004): Selling OR: An Historical perspective, OR/ MS Today vol 31, no5 pg $30-36$

[13] Wayne L.W(1995): Introduction to Mathematical Programming, Applications and algorithms, $2^{\text {nd }}$ edition, Duxbury Press, USA.

[14] William Thomas: $25^{\text {th }}$ Anniversary: History of Operational Research, useful history of OR, vol 42 www.informs.org/ORMS- Today/ public -Articles volume 42 